\title{
FATOR DE CONDIÇÃO GONADAL, ÍNDICE HEPATOSSOMÁTICO E RECRUTAMENTO COMO INDICADORES DO PERÍODO DE REPRODUÇÃO DE LORICARIICHTHYS PLATYMETOPON (OSTEICHTHYES, LORICARIIDAE), BACIA DO RIO URUGUAI MÉDIO, SUL DO BRASIL
}

\author{
Marcus Vinicius Morini Querol ${ }^{1}$ \\ Enrique Querol ${ }^{1}$ \\ Nara Neide Adolpho Gomes ${ }^{1}$
}

\begin{abstract}
GONADAL CONDITION FACTOR, HEPATOSSOMATIC INDEX AND RECRUITMENT AS INDICATORS OF REPRODUCTIVE PERIOD OF LORICARIICHTHYS PLATYMETOPON (OSTEICHTHYES, LORICARIIDAE), MEDIUM URUGUAY RIVER BASIN, SOUTH OF BRAZIL. The recruitment period of Locariichthys platymetopon (Isbrucker \& Nijssen, 1979) was investigated. It has been found out, through the data analysis, that the gonadal condition factor is a good indicator of the reproductive period and that the hepatossomatic index is probably related to the storage of reservation for the winter period. The recruitment occurred in November coinciding with the reproductive activity of May.
\end{abstract}

KEYWORDS. Pisces, Loricariidae, Locariichthys, reproductive, Uruguay river.

\section{INTRODUÇÃO}

A ação antrópica sobre o rio Uruguai, cada vez mais expressiva, destruindo nichos existentes, alterando as populações ícticas e o ecossistema, traz a necessidade de estudos de dinâmica de populações como alternativa que poderá contribuir para a preservação e gerenciamento pesqueiro desta importante bacia (QueRoL et al.,1997).

O cascudo Loricariichthys platymetopon (Isbrucker \& Nijssen, 1979) é uma das espécies de fácil captura e, gradativamente, tem-se observado um aumento na sua comercialização na região de Uruguaiana, Rio Grande do Sul. AzEvedo (1938) referiu-se às espécies de peixes da família Loricariidae, como tendo grandes perspectivas para o cultivo, não só devido ao valor de sua carne como, também, à sua adaptação a ambientes lênticos, e à pouca exigência quanto ao teor de oxigênio, além de serem de cadeia alimentar curta. Estas características, associadas às populações nos hábitats naturais, possibilitam

1. Museu de Ciências, Núcleo de Pesquisas Ictiológicas, Limnológicas e Aquicultura da Bacia do Rio Uruguai (NUPILABRU), Pontifícia Universidade Católica do Rio Grande do Sul, Campus de Uruguaiana, BR 472-Km 07, Caixa Postal 249, 97500-970, Uruguaiana, RS, Brasil. (mquerol@pucrs.campus2.br) 
seu emprego como matrizes, na grande extensão de ambientes lênticos artificiais existente na região de Uruguaiana, permitindo desta forma sua utilização em cultivo semi-intensivo.

Objetiva-se avaliar o fator de condição e o índice hepatossomático como indicadores do período reprodutivo, relacionando-os com o índice gonadossomático e determinar a época de recrutamento de L. platymetopon.

\section{MATERIAL E MÉTODOS}

Foram utilizados 438 exemplares de Loricariichthys platymetopon, dos quais 192 machos e 246 fêmeas, coletados mensalmente durante o período de agosto de 1995 a julho de 1996, em um reservatório com aproximadamente 180 hectares. Este é destinado à irrigação da cultura do arroz e comunica-se à montante e à jusante com a Sanga do Guarapuitan que desemboca no arroio de mesmo nome, percorrendo uma distância aproximada de $12600 \mathrm{~m}$ até o rio Uruguai, na Estância Nova Esperança, localidade de Santana Velha, município de Uruguaiana, fronteira oeste do Rio Grande do Sul (29 $\left.59^{\prime} 12^{\prime \prime} \mathrm{S}-57^{\circ} 12^{\prime} 55^{\prime \prime} \mathrm{W}\right)$.

Para as coletas, utilizaram-se um barco de alumínio, com motor de popa de $8,8 \mathrm{HP}$, e as seguintes artes de pesca: cinco redes de malhas de $1,5 \mathrm{~cm}$ até $5 \mathrm{~cm}$ entre-nós adjacentes, $20 \mathrm{~m}$ a $50 \mathrm{~m}$ de comprimento e $1,5 \mathrm{~m}$ a $3 \mathrm{~m}$ de altura, além de peneiras, para a captura de exemplares de diversos comprimentos. As redes foram colocadas em diferentes locais da barragem por um período de $24 \mathrm{~h}$. Os exemplares foram fixados em formol a $10 \%$, e, no laboratório, medidos, pesados e eviscerados, tendo as gônadas e o fígado separados e conservados em álcool $70 \%$. O material está depositado na coleção científica do Museu da Pontifícia Universidade Católica do Rio Grande do Sul, Campus de Uruguaiana.

Para cada exemplar registraram-se o sexo, comprimento total (Lt) e comprimento padrão (Ls) com precisão de $(0,1 \mathrm{~cm})$, o peso total $(\mathrm{Wt})$, peso das gônadas $(\mathrm{Wg})$ e peso do fígado (Wf), precisão de 0,01 gramas. A determinação do sexo foi realizada pela observação macroscópica das gônodas e do lábio inferior o qual se apresenta, nos machos, com uma borda membranosa abaixo da parte mais espessa do lábio, técnica utilizada por Melo et al. (1995). O período reprodutivo foi estabelecido por meio da análise mensal do fator de condição e do índice hepatossomático, comparandoos com a curva de maturação, com base na variação mensal do índice gonadossomático médio.

Os cálculos do índice gonadossomático (IGS), fator de condição sob a influência do peso das gônadas $(\mathrm{K})$ e sem o peso das gônadas (K'), fator de condição gonadal $(\Delta \mathrm{K})$ e índice hepatossomático (IHS), foram efetuados conforme Vazzoler (1981, 1996): IGS=Wg/Wt.100; IGS, índice gonadossomático; $\mathrm{Wg}$, peso da gônada; $\mathrm{Wt}$, peso total do peixe; $\mathrm{K}=\mathrm{Wt} / \mathrm{Ls}^{\mathrm{b}} ; \mathrm{K}^{\prime}=\mathrm{Wc} / \mathrm{Ls}^{\mathrm{b}}$; $\mathrm{Wc}=$ $\mathrm{Wt}-\mathrm{Wg}$; Wt, peso total (g); Wc, peso total menos o peso da gônada; Ls, comprimento total (mm); $\mathrm{b}$, coeficiente de alometria obtido através da relação peso-comprimento; $\Delta \mathrm{K}=\mathrm{K}-\mathrm{K}^{\prime}$; IHS=Wf/Wt.100; IHS, índice hepatossomático; Wf, peso do fígado.

A época de recrutamento, indicando o período em que novos indivíduos são incorporados à população, foi determinada através da captura de larvas e alevinos de L. platymetopon e observação e captura de larvas no momento da eclosão dos ovos.

\section{RESULTADOS E DISCUSSÃO}

O período de reprodução de Loricariichtys platymetopon, registrado através da análise mensal dos valores médios do fator de condição, índice hepatossomático em função do índice gonadossomático (IGS) das fêmeas (fig. 1) e dos machos (fig. 2), demonstrou variações médias dos valores dos índices dos desvios padrões do IGS de fêmeas e machos adultos (tab. I). Pela análise dos resultados do IGS das fêmeas, o período reprodutivo foi determinado entre os meses de novembro a fevereiro, atingindo o pico de reprodução em novembro, tendo o início do desenvolvimento gonadal ocorrido no decorrer do mês de agosto. $\mathrm{O}$ incremento no IGS em agosto tem sido observado em outras espécies da região, como na traíra Hoplias malabaricus Bloch, 1794 (QUEROL \& 
Tabela I. Variações médias dos valores dos índices dos desvios padrões do índice gonadossomático de fêmeas adultas e machos adultos de Loricariichtys platymetopon, capturados no reservatório da estância Nova Esperança, Santana Velha, Uruguaiana, RS, de agosto/95 a julho/96.

\begin{tabular}{lll}
\hline & Fêmeas & Machos \\
\hline AGO & $\pm 0,477$ & $\pm 0,082$ \\
SET & $\pm 1,494$ & $\pm 0,058$ \\
OUT & $\pm 2,436$ & $\pm 0,091$ \\
NOV & $\pm 3,204$ & $\pm 0,071$ \\
DEZ & $\pm 1,969$ & $\pm 0,091$ \\
JAN & $\pm 1,862$ & $\pm 0,073$ \\
FEV & $\pm 0,414$ & $\pm 0,037$ \\
MAR & $\pm 0,145$ & $\pm 0,028$ \\
ABR & $\pm 0,359$ & $\pm 0,07$ \\
MAI & $\pm 0,428$ & $\pm 0,034$ \\
JUN & $\pm 0,41$ & $\pm 0,053$ \\
JUL & $\pm 0,347$ & $\pm 0,048$ \\
\hline
\end{tabular}

QUEROL, 1993). Este aumento do IGS pode estar relacionado à elevação da temperatura ocorrida nos últimos anos neste período. Destaca-se que, nesse mês e no subseqüente, não foram coletados espécimes maturos de L. platymetopon, larvas e alevinos, o que sugere, apesar da elevação significativa do IGS, não ocorrer desova neste período. Este fato também foi observado quando se analisa o fator de condição gonadal das fêmeas, no qual ocorreu um aumento dos valores de $\Delta \mathrm{K}$ (fig. 3), no período de agosto-setembro, de forma semelhante à encontrada no IGS.

Os resultados obtidos para o IGS são semelhantes aos encontrados por BRUSCHI et al. (1997), que estudaram o período reprodutivo de Loricariichthys anus (Valenciennes, 1840), na lagoa Emboaba, Osório, RS, ocorrendo uma elevação do índice no período, de novembro a março. O pico de desova ocorreu também entre os meses de outubro e novembro. Analisando o fator de condição gonadal $(\Delta \mathrm{K})$, como indicador do período de reprodução das fêmeas de L. platymetopon, observou-se uma elevação nos seus índices a partir de outubro, tendo seu cume em dezembro, quando ocorreu um declínio até fevereiro, de forma semelhante aos resultados de IGS aqui obtidos. Estes dados sugerem que o fator de condição indica que a espécie está fisiologicamente melhor preparada para a reprodução neste período, revelando-se bom indicador do período de reprodução das fêmeas. Analisando o IGS e o $\Delta \mathrm{K}$ das fêmeas, mediante a correlação de Pearson, foi obtido $\mathrm{r}=0,92$, o que permite inferir uma alta correlação entre o fator de condição gonadal e o índice gonadossomático, podendo, desta forma, serem utilizados ambos índices para a determinação do período reprodutivo.

Analisando os valores médios do índice gonadossomático dos machos (fig. 2), observou-se que ocorre, a partir de outubro, um forte aumento nos seus índices, atingindo seu ápice em novembro, mantendo-se aproximadamente estável até dezembro, a partir do qual ocorre uma forte queda até janeiro, diminuindo lentamente até março. Entre março e abril ocorre um incremento que possivelmente não esteja relacionado com a reprodução. Os machos estão aptos para a reprodução de novembro a dezembro, coincidentemente com o período de reprodução das fêmeas. $\mathrm{O}$ fator de condição gonadal $(\Delta \mathrm{K})$, como indicador do período reprodutivo dos machos (fig. 4), revelou resultados semelhantes 


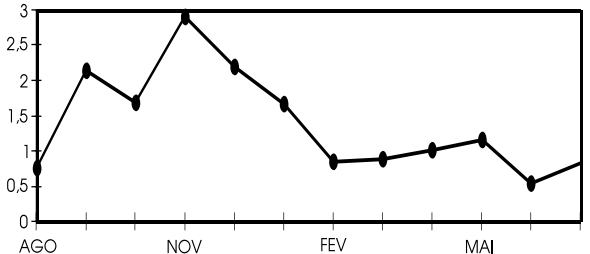

1

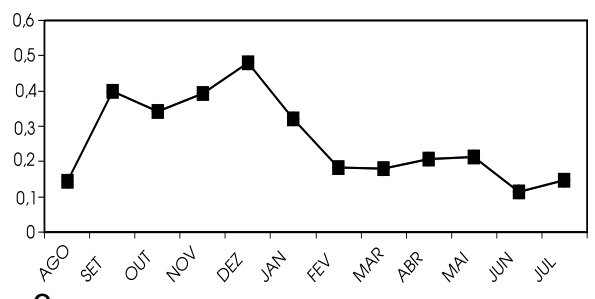

3

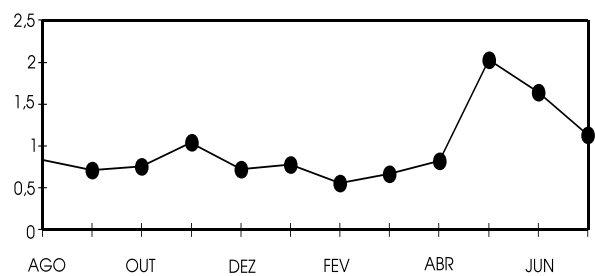

5
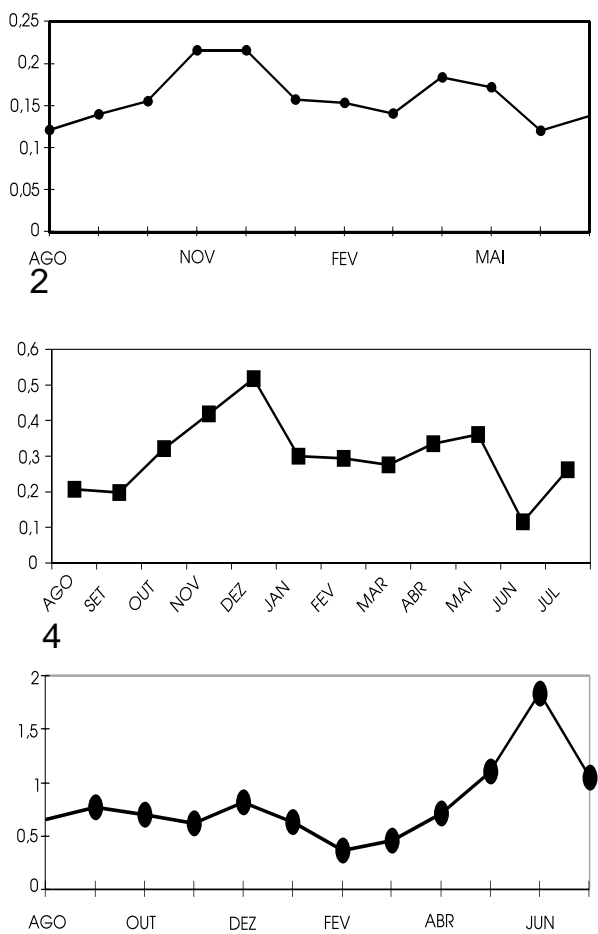

6

Figs. 1-6. Loricariichtys platymetopon, capturados no reservatório da estância Nova Esperança, Santana Velha, Uruguaiana, RS, de agosto/95 a julho/96, fêmeas adultas, n=246 e machos adultos n=192 (valores médios mensais). Índice gonadossomático: 1, fêmeas; 2, machos; fator de condição gonodal: 3, fêmeas; 4, machos; índice hepatossomático: 5, fêmeas; 6, machos.

ao das fêmeas, apresentando também uma elevação nos seus índices a partir de setembro e cume em dezembro, quando diminui até janeiro, sugerindo desta forma, que o fator de condição gonadal, para ambos os sexos, pode ser utilizado como um bom indicador do período reprodutivo de L. platymetopon. Através da análise de correlação entre o IGS e o $\Delta \mathrm{K}$ dos machos, encontramos para $\mathrm{r}=0,92$, o que também permite dizer que existe alta correlação entre o fator de condição gonadal e o índice gonadossomático.

O índice hepatossomático (IHS), para ambos os sexos de L. platymetopon, não pode ser utilizado como indicador do período reprodutivo, pois apresenta seus maiores índices a partir de abril estendendo-se a julho, com elevação do pico em maio para as fêmeas (fig. 5) e em junho para os machos (fig. 6). O IHS parece estar relacionado com o acúmulo de reservas energéticas para o período de inverno. Este fato é evidenciado pela baixa correlação entre o índice hepatossomático e o indice gonadossomático, $\mathrm{r}=-0,21$.

$\mathrm{O}$ fator de condição e o índice hepatossomático têm sido utilizados como indicadores do período reprodutivo, correlacionado a outros fatores como o IGS. O índice hepatossomático pode estar relacionado com a mobilização das reservas energéticas necessária para o processo de vitelogênese, reprodução ou preparação para 
o período de inverno.

Os resultados obtidos para L. platymetopon, em Uruguaiana, são semelhantes aos encontrados por IsAaC-NAHUM \& VAZzOLER (1983), para Micropogonias furnieri (Desmarest, 1823), por BARBIERI \& VERANI (1987) para Hypostomus aff. plecostomus (Linnaeus, 1758), na represa Monjolinho, São Carlos, SP e BARBIERI et al., (1996) para Astyanax fasciatus (Cuvier, 1819), na represa do Lobo, SP, que constataram uma variação do fator de condição associada ao período de reprodução constituindo-se, desta forma, um bom indicador do período de desova.

A época de recrutamento, indicando o período em que novos indivíduos são incorporados à população, ocorreu em novembro, coincidindo com o pico de maior atividade reprodutiva, obtido por meio da análise do IGS para ambos os sexos, quando foram capturados machos de L. platymetopon carregando os ovócitos ventral e externamente sob o lábio inferior, bem como algumas larvas e alevinos coletados mediante o uso de peneiras. Também foi capturado um grande número de larvas de L. platymetopon obtido pela eclosão dos ovos no momento da captura, corroborando o período de postura e indicando a época de recrutamento.

BRUSCHI et al. (1997), citando diferentes autores, afirmaram que várias espécies de Loricariidae desenvolvem estratégia de cuidado parental, tendo observado em $L$. anus um crescimento do aparelho bucal (lábio) dos machos nos meses que antecedem à reprodução, permitindo guardar os ovos aderentes após a desova. Este fenômeno foi registrado para Loricariichthys por Menezes (1949) e MACHADO-AlLison \& LoPEZ-RoJAS (1975) em L. typus (Bleekler, 1864), por TAYLOR (1983) em L. anus e por IsBrUCKER \& NiJSSEN (1979) em L. platymetopon.

Através da análise dos dados, constatou-se que o fator de condição gonadal é um bom indicador do período reprodutivo, enquanto que o índice hepatossomático está provavelmente relacionado ao acúmulo de reservas para o período de inverno. $\mathrm{O}$ recrutamento ocorreu no mês de novembro coincidindo com o pico de maior atividade reprodutiva.

Agradecimentos. Ao Sr. Miguel Augusto Barbara e família pelo acesso ao local de coleta e pelo apoio à NUPILABRU e a esta equipe pela colaboração nas diferentes etapas deste trabalho, à Prof. Gonçalina Alves Simões pela redação do abstract, ao CNPq pela bolsa de mestrado (processo 131390/95-3) e à FAPERGS pelas bolsas (BIC).

\section{REFERÊNCIAS BIBLIOGRÁFICAS}

Azevedo, P. 1938. O cascudo dos açudes nordestinos Plecostomus plecostomus. Publ. Inst. Pesca, Buenos Aires, 10(9):211-224.

Barbieri, G.; Hartz, S. M. \& Verani, J. R. 1996. O fator de condição e o índice hepatossomático como indicadores do período de desova de Astyanax fasciatus da represa do Lobo, São Paulo (Osteichthyes, Characidae). Iheringia, Sér. Zool., Porto Alegre, (81):97-100.

Barbieri, G. \& Verani, J. R. 1987. O fator de condição como indicador do período de desova em Hypostomus aff. plecostomus (Linnaeus, 1758) (Osteichthyes, Loricariidae), na represa do Monjolinho, São Carlos, SP. Ciênc. Cult., São Paulo, 39(7):655-658.

Bruschi, W., Jr.; Peret, A. C. et al. 1997. Reprodução de Loricariichthys anus (Valenciennes, 1840) da Lagoa Emboaba, Osório, RS. Revta bras. Biol., Rio de Janeiro, 57(4):677-685.

IsaAc-Nahum, V. J. \& VAzzoler, A. E. A. DE M. 1983. Biologia reprodutiva de Micropogonias furnieri (Desmarest, 1823) (Teleostei, Sciaaenidae), 1. Fator de condição como indicador do período de desova. Bolm Inst. Oceanogr., São Paulo, 32(1):63-69. 
IsBrucker, I. J. H. \& NiJssen, H. 1979. Three new south american mailed catfishes of the genera Rineloricaria and Loricariichthys (Pisces, Siluriformes, Loricariidae). Bijdr. Dierk., Amsterdam, 48(2):151-211.

Machado-Allison, H. \& Lopez-Rojas, A. 1975. Etapas del desarollo de Loricariichthys typus (Bleeker, 1864) (Osteichthyes, Siluriformes, Loricariidae). Acta Biol. venez., Caracas, 9:93-119.

Melo, J. F. DE; Querol, M. V. M. et al. 1995. Dados preliminares sobre a biologia e reprodução do cascudo viola Loricariichthys anus (Pisces, Loricariidae), na região de Uruguaiana, RS, Brasil. Hífen, Uruguaiana, 19(35/36):34-37.

Menezes, R. S. DE 1949. Incubação labial de ovos pelo macho de Loricaria typus Bleeker, da lagoa do peixe, Piauí, Brasil, (Actinopterygii, Loricaridae, Loricariinae). Revta bras. Biol., Rio de Janeiro, 9(3):381-387.

Querol, E; Querol, M. V. M. \& Lobón-Cerviá, J. 1997. Estimativa da densidade e biomassa da população de Cichlasoma portalegrense (Hensel, 1870) (Pisces, Cichlidae) através do método de três capturas sucessivas com pesca elétrica em um arroio do pampa brasileiro. Comun. Mus. Ciênc. PUCRS, Sér. Zool., Porto Alegre, 10:13-25.

Querol, M. V. M. \& Querol, E. 1993. Reprodução de traíra Hoplias malabaricus (Pisces, Erythrynidae) na região de Uruguaiana, Rio Grande do Sul, Brasil. Hífen, Uruguaiana, 18(34):31-45.

TAYLOR, J. N. 1983. Field observations on the reproductive ecology of three species of armored catfishes (Loricariidae: Loricariinae) in Paraguay. Copeia, Austin, 1983(1):257-259.

Vazzoler, A. E. A. DE M. 1981. Manual de métodos para estudos biológicos de população de peixes. Reprodução e crescimento. Brasilia, CNPq - Programa Nacional de Zoologia. $108 \mathrm{p}$.

_. 1996. Biologia da reprodução de peixes teleósteos: teoria e prática. Maringá, EDUEM. $169 \mathrm{p}$.

Recebido em 08.08.2001; aceito em 03.07.2002. 